\title{
Combined treatment of surgery and sclerotherapy for sinus pericranii
}

\author{
Jeong Yeop Ryu ${ }^{1}$, \\ Jong Ho Lee', \\ Joon Seok Lee ${ }^{1}$, \\ Jeong Woo Lee ${ }^{1}$, \\ Seok Jong Lee ${ }^{2}$, \\ Jong Min Lee, \\ Sang Yub Lee, \\ Seung $\mathrm{Huh}^{4}$, \\ Ji Yoon Kim ${ }^{5}$, \\ Sung Kyoo Hwang ${ }^{6}$, \\ Ho Yun Chung ${ }^{1,7}$ \\ Departments of ${ }^{1}$ Plastic and \\ Reconstructive Surgery, ${ }^{2}$ Dermatology, \\ ${ }^{3}$ Radiology, ${ }^{4}$ Surgery, ${ }^{5}$ Pediatrics, and \\ ${ }^{6}$ Neurosurgery and ${ }^{7}$ Cell \& Matrix \\ Research Institute, School of Medicine, \\ Kyungpook National University, Daegu, \\ Korea
}

\begin{abstract}
Sinus pericranii is a rare vascular anomaly characterized by abnormal venous communication between the inner and outer regions of the cranial cavity. Here, we report a case of sinus pericranii and venous malformations in the right periorbital region of a 2-year-old girl. Radiologic findings showed venous malformations in the right parietal region communicating with the superior sagittal sinus in the intracranial region. There were notable improvements following surgical resection for the abnormal venous lesions and several sclerotherapies. Presence of a bluish and pulsating mass on the scalp, which showed bruit on auscultation, may indicate sinus pericranii, which should be included in the differential diagnosis.
\end{abstract}

Keywords: Head / Plastic / Sclerotherapy / Sinus pericranii / Surgery / Vascular malformations

\section{INTRODUCTION}

Sinus pericranii (SP) is a rare venous anomaly characterized by an abnormal communication between the intracranial and extracranial veins. Pathologically, it exhibits a direct communication between the nonmuscular veins adhering to the outer surface of the skull and the intracranial venous sinuses [1]. There are several etiologies for SP, such as congenital, spontaneous, and posttraumatic [2]. SP patients may present with mild (local pain, nausea, headache, and dizziness) to severe (increased intracranial pressure, bradypnea, bradycardia, ataxia, hearing loss,

\section{Correspondence: Ho Yun Chung}

Department of Plastic and Reconstructive Surgery, School of Medicine, Kyungpook National University, 130 Dongdeok-ro, Jung-gu, Daegu 41944, Korea E-mail: hy-chung@knu.ac.kr

This work was supported by the National Research Foundation of Korea (NRF) grant funded by the Korea government (MSIT) (No. 2020R1A2C2009496).

Received September 25, 2019 / Revised October 22, 2019 / Accepted October 28, 2019 and epileptic seizures) symptoms [3]. However, most cases are asymptomatic, with the main complaint being the associated cosmetic problems [4]. Additionally, considering its clinical presentation, it is imperative to identify SP from other diseases presenting as a mass on the scalp. Here, we report a clinical case of SP, with the diagnosis and treatment.

\section{CASE REPORT}

\section{Patient information}

A 2-year-old girl was brought to the hospital due to an apparent disfigurement and bluish skin in the right periorbital region and a soft mass-like diffuse lesion on the scalp. The lesion was non-pulsatile and bluish, and the patient did not complain of pain. There was no relevant history, and she was delivered vaginally after a full-term pregnancy (Fig. 1). 

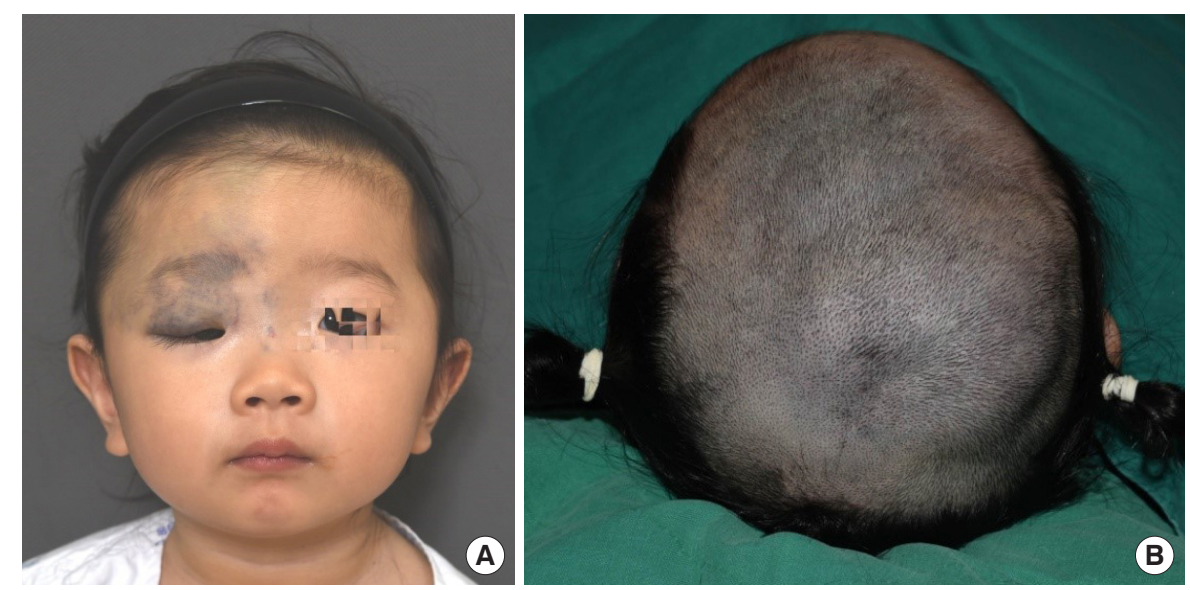

Fig. 1. A 2-year-old girl with sinus pericranii. (A) Venous malformations in the right periorbital region are seen on the frontal view. (B) Vascular masses on the scalp are also seen.
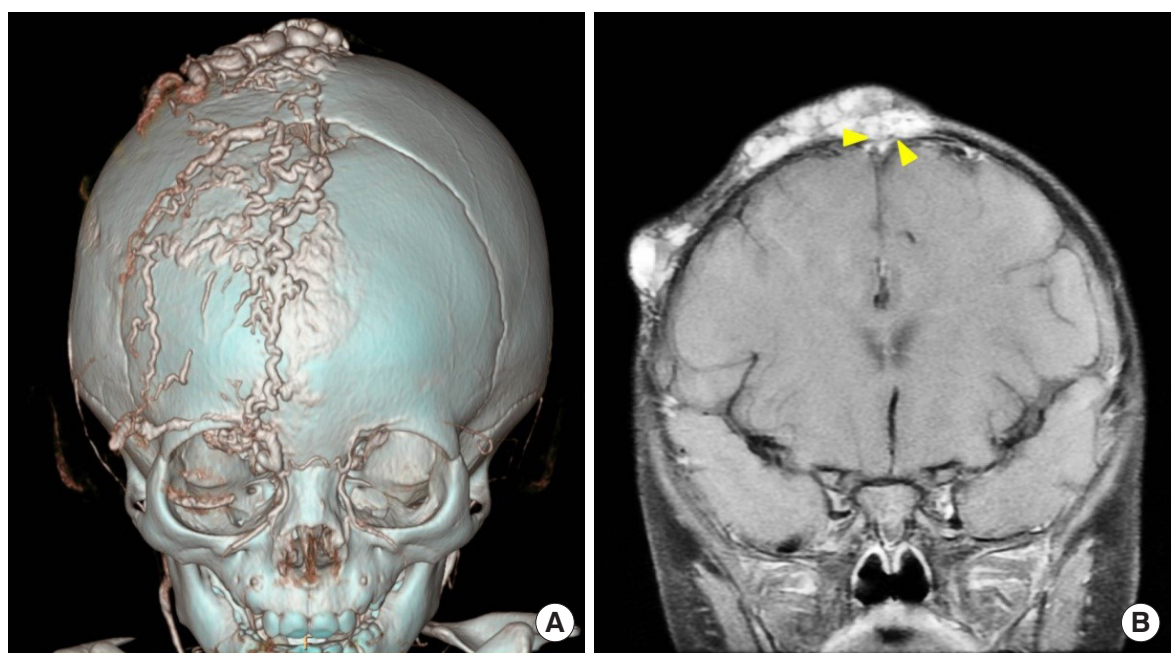

Fig. 2. Radiologic images of the patient. (A) Venous malformations in the scalp and periorbital region are observed on computed tomography angiography. (B) The lesion in the scalp is suspected to communicate with the superior sagittal sinus (yellow arrowheads) on magnetic resonance imaging.

\section{Image procedures}

Computed tomography confirmed vascular masses in the right periorbital, retrobulbar, and extracranial parietal regions. Doppler ultrasonography and magnetic resonance imaging (MRI) confirmed venous malformations in the aforementioned regions (Fig. 2). We suspected the presence of cranial holes facilitating the communication between the extracranial venous malformations and intracranial superior sagittal sinus, as observed on MRI. Therefore, we performed a transfemoral cerebral angiography (TFCA) to confirm the presence/absence of holes. Visualizing the right internal carotid artery via angiography revealed transdural and transcranial superficial drainage from the frontal cortical veins and from the superior ophthalmic veins. Therefore, we confirmed that subcutaneous venous malformations in the scalp communicated with the superior sagittal sinus and diagnosed the patient with SP.

\section{Surgical treatment}

After placing the patient in the supine position under general anesthesia, a plastic surgeon and neurosurgeon made an incision in the scalp on the left side. After confirming engorged veins in the subgaleal layer by performing a slight periosteal dissection of the scalp from the left to the right side, we ligated and excised the abnormal extracranial veins. Following which, we ligated and removed the veins draining into the superior sagittal sinus, through a relatively large cranial hole in the midline. The cranial hole was sealed with bone wax. We consequently created a subgaleal negative drainage and ended the surgery by suturing the wound (Fig. 3). 

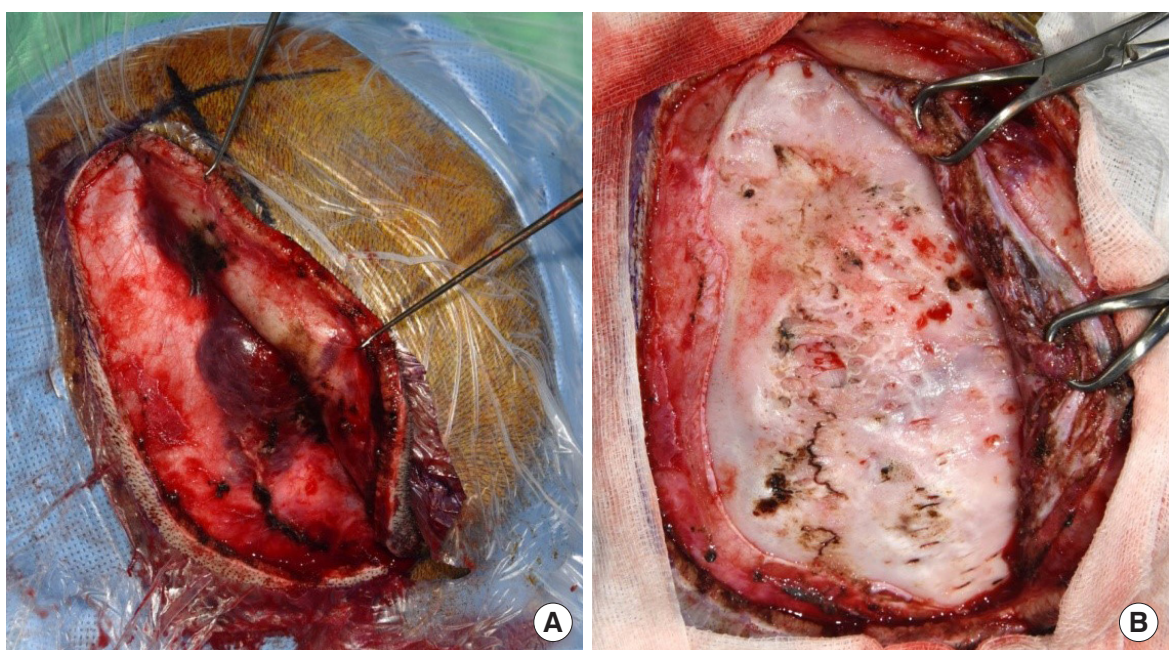

Fig. 3. Intraoperative views of sinus pericranii. (A) Huge venous engorgement on the extracranial scalp is seen. (B) After resection of venous malformations, small cranial holes communicating with the intracranial region are seen. The veins are ligated and obliterated with electrocautery and bone wax.

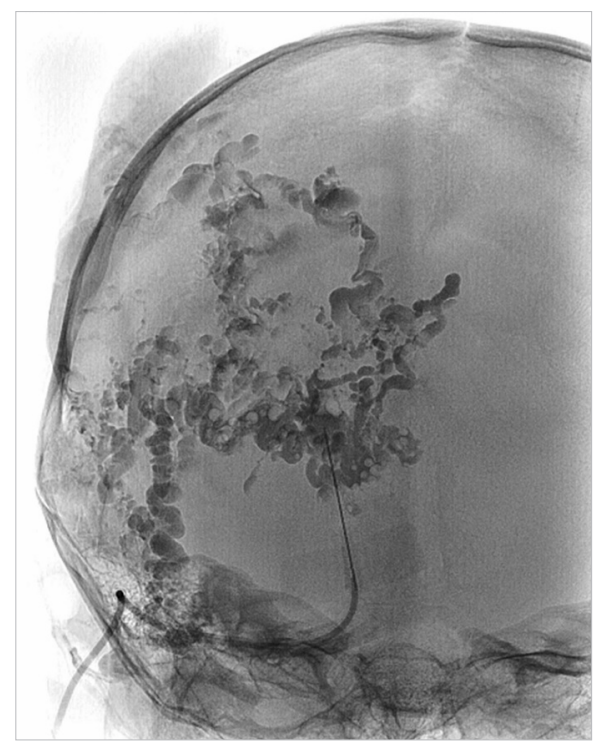

Fig. 4. Sclerotherapy for sinus pericranii. After ultrasonographyguided needling, sclerotherapy is being performed using a $30-\mathrm{mL}$ mixture containing bleomycin, without acute complications.

\section{Sclerotherapy}

A radiology specialist performed four sessions of sclerotherapy on the patient under general anesthesia, after administering bleomycin via a butterfly needle after ultrasonography-guided needling for the right superior ophthalmic venous malformations and for SP in the right parietotemporal scalp region (Fig. 4). Follow-up ultrasonography and MRI showed marked improvements in venous malformations and SP.

\section{Follow-up and outcomes}

In the postoperative course, the mass on the scalp greatly de-

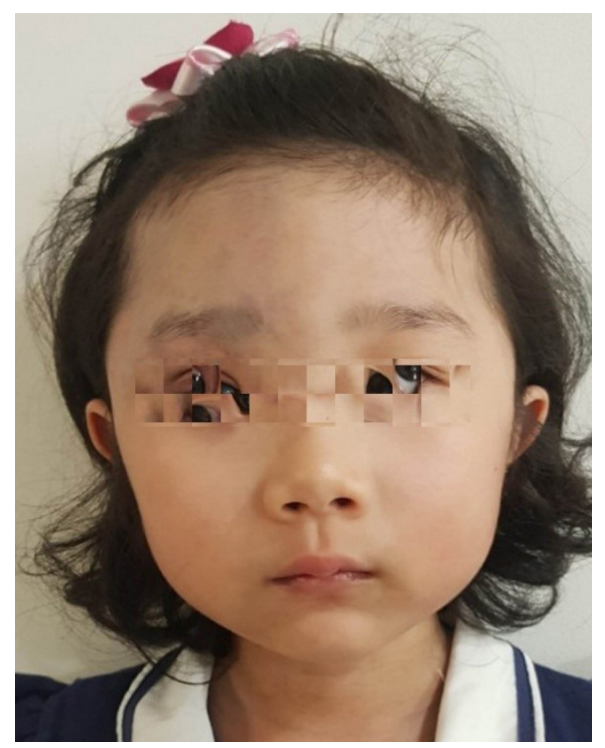

Fig. 5. Photograph of the patient 33 months after surgery. After surgical resection and sclerotherapies, venous malformations in the scalp and periorbital area have markedly improved.

creased in volume, and the abnormally communicating veins disappeared. We subsequently performed another excision and six sessions of sclerotherapy using bleomycin on the remaining venous malformations in the periorbital region. Currently, the patient is under observation for the duration of the follow-up period (Fig. 5).

\section{DISCUSSION}

SP is a rare venous anomaly that includes emissary diploic veins originating from the intracranial sinus and leading to a subga- 
leal drainage, and forms a varix on the outer surface of the skull [5]. This anomaly can develop independently or concomitantly with other malformations, including dural sinus hypoplasia or non-syndromic or syndromic craniosynostosis [6-8]. Although its etiologies have not been identified, it strongly correlates with those of intracranial venous anomalies [3]. Reportedly, SP can also be caused by trauma.

Most SP patients visit the hospital primarily complaining of the mass on the scalp. However, excisions similar to those performed for benign masses on the scalp may prove hazardous because of the communicating venous anomalies. Therefore, it is imperative to eliminate SP from the differential diagnoses before excising the mass. Since it is a venous anomaly, it can be diagnosed with methods similar to those used for other venous anomalies in the head and neck region. Doppler ultrasonography is a useful radiological tool to differentiate different vascular anomalies. Following which, MRI can be used to characterize the size and extent of the lesion. T2-weighted MRI shows a mass with high levels of mixed signals, which includes a signal void due to the blood flow [9]. In the present patient, a dilated venous space was observed on Doppler ultrasonography, and SP was diagnosed owing to venous malformations directly draining into the major veins. MRI revealed a lesion with venous anomalies that were suspected to be communicating with the superior sagittal sinus, following which we performed TFCA. TFCA confirmed the communication of venous malformations with extracranial venous malformations and superior sagittal sinus.

First, we performed sclerotherapy to treat venous malformations in the periorbital region. We selected a noninvasive procedure to avoid damaging the vital adjacent structures. Considering the patient's age, a radiologist performed ultrasonographyguided needling, and an anesthesiologist performed sclerotherapy using bleomycin under general anesthesia. Bleomycin is reportedly a safe drug in sclerotherapy, with short-term effects, a good degree of patient satisfaction, and no long-term side effects $[10,11]$. There was a notable improvement in the lesion following sclerotherapy, and had significantly reduced in size on the follow-up imaging outcomes. We surgically removed the lesion on the scalp in a procedure performed by plastic surgeons and neurosurgeons. Some reports suggest that surgical excisions for SP could be considered in the presence of neurologic symptoms, psychological discomforts, dermatological changes, patient's wishes, and coexisting morbidity. Additionally, coexisting morbidities included syndromic craniosynostosis, additional vascular anomalies, meningocerebral anomalies, and systemic anomalies or malformations [3,4]. Despite the absence of symptoms during diagnosis, it is possible that the patient may develop mild to severe symptoms with age; however, the
Table 1. Previous reports on the treatment of sinus pericranii

\begin{tabular}{lrl}
\hline Study & No. of patients & \multicolumn{1}{c}{ Treatment } \\
\hline Aburto-Murrieta et al. [2] & 1 & Conservative treatment \\
Pavanello et al. [3] & 21 & Surgery and endovascular embolization \\
Shaw et al. [8] & 1 & Surgery \\
Tamura et al. [12] & 1 & Surgery \\
Ito et al. [13] & 1 & Surgery \\
Ohshima et al. [14] & 1 & Endovascular embolization \\
\hline
\end{tabular}

patient's parents wished to treat the lesion considering the apparent cosmetic problems. Moreover, the mass seemed to increasing in size over time and presence of additional venous malformations in the periorbital region was also considered to surgically treat the SP. Surgical treatment involved removing extracranial venous anomalies and ligating the communicating emissary vein. Multiple erosions were observed in the bone because of venous malformations, which further confirmed the presence of an abnormal communication. These erosions were subsequently treated with bone wax. We postoperatively performed sclerotherapy on the residual venous malformations in the scalp using bleomycin. Preoperative sclerotherapy was avoided to prevent the drainage of the sclerotic agent into the intracranial sinus due to the communication with the intracranial sinus. This communication was eliminated perioperatively, following which we performed sclerotherapy. The lesion associated with SP disappeared. The patient is on follow-up for observation of venous anomalies in the periorbital region and the need for any form of additional treatment.

Previous reports on SP included surgical treatment, endovascular embolization, and conservative treatment (Table 1) [2,3,8, $12-14]$. However, it is difficult to completely eliminate tiny residual venous malformations with surgical treatment alone, and in cases of residual or new lesions, the surgical treatment has complications due to the requirement of multiple scalp incisions [12]. Furthermore, although embolization alone may block the communication between venous malformations in the pericranium and the intracranial sinus, the direct effect on the endothelial cells with venous malformations is limited. Additionally, embolization has a risk for development of new venous malformations in regions surrounding the existing lesion, and the glue used during embolization may enter the intracranial sinus. However, sclerotherapy using bleomycin works directly on the endothelial cells with venous malformations and diminishes the cells and tissues [15,16]. To date, cases of SP treated with both surgery and sclerotherapy have not been reported.

In conclusion, masses on the scalp that can be clinically palpated, and are easily deformed and differentiated include cavernous angioma, arteriovenous malformation, sebaceous cyst, 
dermoid cyst, and encephalocele. SP should be considered if a mass on the scalp appears bluish and pulsatile and bruit is detected on auscultation, despite its rarity and further work-up is required. It is important to perform a surgical excision after radiological imaging and to ligate the abnormal communicating emissary veins between the intracranial sinus and extracranial venous engorgement. With sclerotherapy and surgical treatment, we treated the residual and recurring lesions with greater precision and achieved positive outcomes.

\section{NOTES}

\section{Conflict of interest}

No potential conflict of interest relevant to this article was reported.

\section{Ethical approval}

The study was approved by the Institutional Review Board of Kyungpook National University Hospital (IRB No. 2018-10010) and performed in accordance with the principles of the Declaration of Helsinki. Written informed consent was obtained.

\section{Patient consent}

The patient's parent provided written informed consent for the publication and the use of her images.

\section{ORCID}

Jeong Yeop Ryu https://orcid.org/0000-0003-2812-5051

Jong Ho Lee https://orcid.org/0000-0002-8318-5404

Joon Seok Lee https://orcid.org/0000-0002-1580-0487

Jeong Woo Lee https://orcid.org/0000-0003-4903-6066

Seok Jong Lee https://orcid.org/0000-0002-6131-632X

Jong Min Lee https://orcid.org/0000-0002-4163-913X

Sang Yub Lee https://orcid.org/0000-0001-8529-8229

Seung Huh https://orcid.org/0000-0002-0275-4960

Ji Yoon Kim https://orcid.org/0000-0002-5577-6629

Sung Kyoo Hwang https://orcid.org/0000-0003-0146-5232

Ho Yun Chung https://orcid.org/0000-0001-7359-3044

\section{REFERENCES}

1. Amuluru K, Al-Mufti F, Shah M, Prestigiacomo CJ, Liu JK, Gandhi CD. Acquired sinus pericranii due to parasagittal meningiomatous invasion of the superior sagittal sinus and bilateral transverse sinuses. World Neurosurg 2017;98:872.

2. Aburto-Murrieta Y, Bonifacio-Delgadillo D, Balderrama Banares J, Zenteno Castellanos MA. Sinus pericranii: case re- port. Vasc Endovascular Surg 2011;45:103-5.

3. Pavanello M, Melloni I, Antichi E, Severino M, Ravegnani M, Piatelli G, et al. Sinus pericranii: diagnosis and management in 21 pediatric patients. J Neurosurg Pediatr 2015;15:60-70.

4. Gandolfo C, Krings T, Alvarez H, Ozanne A, Schaaf M, Baccin $\mathrm{CE}$, et al. Sinus pericranii: diagnostic and therapeutic considerations in 15 patients. Neuroradiology 2007;49:505-14.

5. Ota T, Waga S, Handa H, Nishimura S, Mitani T. Sinus pericranii. J Neurosurg 1975;42:704-12.

6. Mitsukawa N, Satoh K, Hayashi T, Furukawa Y, Suse T, Uemura $\mathrm{T}$, et al. Sinus pericranii associated with craniosynostosis. J Craniofac Surg 2007;18:78-84.

7. Yasuda S, Enomoto T, Yamada Y, Nose T, Iwasaki N. Crouzon disease associated with sinus pericranii: a report on identical twin sisters. Childs Nerv Syst 1993;9:119-22.

8. Shaw AV, Jayamohan J, Sheerin F, Johnson D. Staged raising of a coronal flap for fronto-orbital advancement and remodeling in saethre-chotzen syndrome complicated by sinus pericranii. J Craniofac Surg 2018;29:1956-9.

9. Ryu JY, Lee JS, Lee JW, Choi KY, Yang JD, Cho BC, et al. Clinical approaches to vascular anomalies of the lip. Arch Plast Surg 2015;42:709-15.

10. Horbach SER, van de Ven JS, Nieuwkerk PT, Spuls PI, van der Horst CMAM, Reekers JA. Patient-reported outcomes of bleomycin sclerotherapy for low-flow vascular malformations and predictors of improvement. Cardiovasc Intervent Radiol 2018; 41:1494-504.

11. Mack JM, Richter GT, Becton D, Salem O, Hill SEM, Crary SE. Short-term side effects and patient-reported outcomes of bleomycin sclerotherapy in vascular malformations. Pediatr Blood Cancer 2018;65:e27008.

12. Tamura G, Ogiwara H, Morota N. Characteristics of recurrent congenital sinus pericranii: case report and review of the literature. Pediatr Neurosurg 2019;54:265-9.

13. Ito E, Takasu S, Hattori K. Sinus pericranii with dominant venous outflow in the superior eyelid. Neurol Med Chir (Tokyo) 2017;57:144-8.

14. Ohshima T, Miyachi S, Joko M, Matsuo N, Kawaguchi R, Maejima R, et al. Endovascular embolization of sinus pericranii using plastic cup during glue injection. World Neurosurg 2019: 124;93-7.

15. Chen Y, Choi CJ, Lee BW. Bleomycin sclerotherapy of a distensible orbital venous malformation without image guidance. Can J Ophthalmol 2019;54:e120-3.

16. Spence J, Krings T, TerBrugge KG, Agid R. Percutaneous treatment of facial venous malformations: a matched comparison of alcohol and bleomycin sclerotherapy. Head Neck 2011;33: 125-30. 